\title{
Centres of evidence-based nursing: directions and challenges
}

Dramatic changes in healthcare delivery, along with an increased emphasis on quality care and cost containment, have led to the need for reliable, up to date evidence about effective healthcare interventions on the part of clinicians, policy makers, consumers, and researchers. However, barriers to using evidence exist for all groups and include those related to the individual, organisation, environment, and the characteristics of the innovation. ${ }^{1}$ For nurses specifically, lack of time, poor access to the literature, and lack of ability to judge the quality of the research are major barriers. ${ }^{23}$ To address these barriers and to facilitate evidence-based practice, several evidencebased nursing centres havebeen created worldwide. In this editorial, we willhighlight the activities of these centres and challenges they face.

We sent a brief questionnaire by email to evidence-based nursing centres in Australasia (Joanna Briggs Institute [JBI], which includes several sites in Australia, New Zealand, and Hong Kong), United States, Germany, UK, and Canada. For the JBI, we contacted the coordinating centre in Adelaide, as well as centres in New Zealand and Victoria. The Victorian Centre of Joanna Briggs is incorporated within the Victorian Centre for Nursing Practice Research. Below, we summarise the information provided by the centres.

\section{Purpose and activities of the centres}

All of the centres have a statement of purpose that relates to improvement of nursing practice and healthcare outcomes through the use of evidence. Although similarities and differences exist in the strategies used by the various centres, most prepare systematic reviews and conduct workshops.

\section{SYSTEMATIC REVIEWS}

Each centre has done or plans to do systematic reviews related to nursing practice. The UK centre, the longest established, has been the most productive in this area, with completed reviews of compression treatment for venous leg ulcers, laser treatment for venous leg ulcers, pressure relieving beds, mattresses and cushions for prevention of pressure sores, the effectiveness of clinical practice guidelines, and support for carers of people with Alzheimer's type dementia. In addition, they have completed preliminary identification and appraisal of randomised controlled trials in nursing. Other reviews in progress at the UK centre include dressings in the treatment of venous leg ulcers, prevention and treatment of diabetic foot ulcers, antibiotics and antimicrobials for chronic wounds, debridement for chronic wounds, electrical stimulation for pressure sores, electrical stimulation for venous leg ulcers, therapeutic ultrasound for pressure sores, therapeutic ultrasound for venous leg ulcers, and wound dressings for pressure sores.

The JBI has recently completed reviews related to falls in acute hospitals, and strategies for preventing and treating chemotherapy and radiation induced oral mucositis in patients with cancer. It is currently completing reviews on vital signs, management of confusion, and group versus individual therapy for people with enduring mental health problems.
The Canadian centre has completed a review related to research dissemination and utilisation, and, in conjunction with the Effective Public Health Practice Project, has been involved in the production of reviews related to adolescent pregnancy primary prevention programmes, home visiting as a delivery strategy for public health nursing interventions to clients in the prenatal and postnatal period, school based suicide prevention programmes for adolescents, primary prevention programmes to prevent sexually transmitted diseases in adolescents, and community interventions to increase fruit and vegetable consumption. The New Zealand centre has 3 reviews in draft form: the instillation of saline bolus in the management of adults with artificial airways, suctioning of patients with artificial airways, and pre- and post-suctioning oxygenation and hyperinflation in patients with artificial airways. The Victorian centre and the German centre have not yet completed any reviews, but plan to promote best practice in the management of incontinence and in the management of breastfeeding infants with failure to thrive (Victorian); and hospital in the home and mobile rehabilitation (Germany).

The Sarah Cole Hirsh Institute (SCHI) in the US has focused its attention on childbearing, childrearing, and caregiving research. Their first "state of the evidence" paper, which summarises US based research with implications for practice, will be released this autumn.

\section{WORKSHOPS}

In addition to reviews, every evidence-based nursing centre conducts local, national, and international workshops on evidence-based nursing: what it is, how to practise it, and how to teach it. Each centre also offers workshops on the implementation of findings from systematic reviews, and most offer "how to" workshops on critical appraisal and the conduct of systematic reviews. Workshops are tailored to meet the needs of the audience, which may include front line clinicians, managers, policy makers, or researchers. Several centres are beginning to consider courses for consumers as well. Courses and workshops on the appraisal and application of systematic reviews are directed towards increasing nurses' and managers' comfort with reading and using systematic reviews. The "how to" of systematic reviews will increase the capacity of nurses and other practitioners to carry out systematic reviews, whereas the workshops on "how to teach $\mathrm{EBN}$ " have the potential to change the ways in which nurses view clinical problems.

\section{OTHER ACTIVITIES}

Each centre is involved in several dissemination strategies using various formats including websites, newsletters, journals, and presentations. Most centres have a strategy for eliciting the views of clinicians about priority topics for systematic reviews and have begun to grapple with issues of how to evaluate the success of implementation for changes in practice or patient outcomes. The New Zealand group is active in the guidelines movement through the New Zealand Guidelines group. 


\begin{tabular}{|c|c|}
\hline Centre & Contact information \\
\hline Joanna Briggs Institute (JBI) for Evidence Based Nursing and Midwifery & $\begin{array}{l}\text { Rick Wiechula, Deputy Director } \\
\text { http://www.joannabriggs.edu.au }\end{array}$ \\
\hline New Zealand Centre for Evidence Based Nursing (collaborating centre of JBI) & $\begin{array}{l}\text { John McArthur, Director } \\
\text { email: johnmc@ahsl.co.nz }\end{array}$ \\
\hline Victorian Centre for Nursing Practice Research (collaborating centre of JBI) & $\begin{array}{l}\text { Linda Johnston, Associate Director and Senior Research Fellow } \\
\text { email: johnstol@cryptic.roh.unimelb.edu.au }\end{array}$ \\
\hline Sarah Cole Hirsh Institute for Best Nursing Practices Based on Evidence & $\begin{array}{l}\text { JoAnne Youngblut, Director } \\
\text { email: jmy3@po.cwru.edu }\end{array}$ \\
\hline German Centre of Evidence Based Nursing & $\begin{array}{l}\text { Johann Behrens } \\
\text { email:Johann.Behrens@medizin.uni-halle.de }\end{array}$ \\
\hline UK Centre for Evidence Based Nursing & $\begin{array}{l}\text { Nicky Cullum } \\
\text { email: nac2@york.ac.uk }\end{array}$ \\
\hline Canadian Centre for Evidence-Based Nursing & $\begin{array}{l}\text { Donna Ciliska, Alba DiCenso, Co-Directors } \\
\text { email: ciliska@fhs.mcmaster.ca or dicensoa@fhs.mcmaster.ca }\end{array}$ \\
\hline
\end{tabular}

The JBI completes audits before and after implementation for each area of clinical practice that is reviewed and distilled into best practice information sheets (based on systematic reviews of the research evidence). The first phase of the evaluation cycle involves documenting outcomes related to a given area of practice in various survey sites. Secondly, recommendations from the best practice information sheets are implemented throughout the survey sites. The final stage involves surveying all sites for changes in practice and patient outcomes. In this way, the JBI centres are evaluating the effects of the information sheets.

\section{Challenges}

There are many challenges in the development and administration of evidence-based nursing centres.

\section{FUNDING}

The SCHI has been very successful in overcoming the funding challenge with a US $\$ 4.4 \mathrm{~m}$ bequest that established the centre. For the other centres, however, the primary challenge is to secure funding for both the infrastructure and various centre activities, such as reviews and workshops. Although some centres rely on university or institutional donations or government grants, many are also trying to recover costs through educational workshops. In some jurisdictions, primary research still has priority over syntheses, with potential funders showing a lack of understanding of why literature reviews cost so much money or how they fit into a "research" agenda; thus, it is sometimes difficult to get project support for synthesis activities.

\section{IDENTIFICATION AND EVALUATION OF DISSEMINATION} STRATEGIES

The second challenge faced by all the centres relates to implementation and evaluation of dissemination strategies. Dissemination and utilisation studies are quite rare in nursing, and when done in other health disciplines are often conducted within a context that is not congruent to nursing practice (eg, academic detailing or use of opinion leaders). Factors related to the innovation, individual, organisation, and environment, and interactions among various factors may hamper the generalisability of findings from one dissemination and utilisation study to another. Ultimately, the centres will need to show that their activities have a measurable effect on processes of care and health outcomes. COORDINATION

Coordination of activities across these international centres is desirable to avoid duplication of activities. Although some review topics may be sensitive to political, cultural, and geographic differences (eg, organisation of care, care delivery, and quality of care reviews), others may cross these boundaries (eg, wound care and catheter care). There is a potential, with the ease of electronic communication, for meaningful collaboration among centres in the conduct of systematic reviews and in the dissemination of the reviews to different groups around the world.

Another challenge for all centres, which could usefully be shared, is how to involve all levels of potential consumers of their products, from clinicians, managers, and policy makers to the general public. As a first step, most centres have solicited input from clinicians by asking for relevant clinical questions to review; this, however, has been the extent of involvement. If we want to reach other target audiences, their involvement is critical to ensure that we are addressing areas of high priority for them.

\section{CULTURE}

The issue of nursing culture in relation to the value and use of evidence is a challenge that is not unique to evidencebased nursing centres. It is, however, one that must be continuously addressed by the centres if they are to have a positive effect. For the most part, nurses are not in the habit of asking clinical questions or searching out answers in the research literature. In one particular study, a group of Canadian nurses identified journals as "the one most common source from which you learn about research findings"; however, the primary journals that the nurses identified were not research journals, but trade magazines. ${ }^{4}$ Estabrooks concluded that traditional scientific journals are not very effective as dissemination vehicles. Indeed, articles published in nursing research journals are more often written for other researchers in a language that is uninterpretable by most clinicians. The evidence-based nursing centres need to translate research results into appropriate messages for the diverse audiences they serve so that high quality information that resides in the centres, the US Agency for Health Care Policy and Research, and the Cochrane Library is understandable and can be used for practice and decision making. However, better readability is only one small step in utilisation. Leaders and managers can also contribute to the creation and support of a culture of evidence-based decision making ${ }^{5}$ by supporting practitioners in their use of the best evidence in the delivery of care. Support here may mean actual working time freed up to go the library, to search for relevant articles, or to organise a journal club. Reliance on doing things the way they have always been done, and the additional energy and resources needed to change practice, mitigate against the likelihood of any such change. Nurses in clinical settings must work hard to be role models of inquiring, critical clinicians who seek answers in the research literature. Cultural change is slow, but awareness of the existence, purpose, and use of the prod ucts and services of the EBN centres may help to promote and support the desired culture change. 
This editorial has provided a brief introduction to various centres for evidence-based nursing to inform our readers of the activities and challenges of the centres. We invite readers to write to us about other centres, to ask questions about the centres, and to suggest alternate activities or strategies for the centres that would facilitate evidence-based nursing practice. Please see below for instructions for letters to the editors. We will be happy to respond to your comments.

DONNA CILISKA, RN, PhD ALBA DiCENSO, RN, PhD

School of Nursing,

Faculty of Health Sciences,

McMaster University,

Hamilton, Ontario, Canada
Centre for Evidence Based Nursing,

Department of Health Studies,

University of York,

York, UK

1 Dobbins M, Ciliska D, DiCenso A. Dissemination and use of research evidence for policy and practice: a framework for developing, implementing and evaluating strategies. A working paper prepare
Nurses Association,1998.(http://www.cna-nurses.ca)

2 Blythe J, Royle JO. Assessing nurses' information needs in the work environment. Bull Med Libr Assoc 1993;81:433-5.

3 Pearcey PA. Achieving research-based nursing practice. $J$ Adv Nurs 1995;22:33-9.

4 Estabrooks CA. Will evidence-based nursing practice make practice perfect? Can J Nurs Res 1998;30:15-36.

5 Evidence-Based Decision Making Working Group. Creating a culture of evidence-based decision making in health. Ottawa: National Forum on Health, 1997.

\section{Acknowledgments}

A s our second year of publication draws to a close, we would like to thank the many people who have contributed - 1 their time, expertise, and enthusiasm to Evidence-Based Nursing.

$\mathrm{T}$ o our panel of advisers on qualitative research, who helped to select qualitative research papers, edited abstracts and commentaries for selected files*, and who continue to help us to develop our approach to abstracting qualitative research:

*Caroline Carlisle, Liverpool, UK

*Marlene Z Cohen, Houston, Texas, USA

Marilyn Ford-Gilboe, London, Ontario, Canada

Jenny Ploeg, Hamilton, Ontario, Canada

*Rita Schreiber, Victoria, British Columbia, Canada

*Carl Thompson, York, UK

*Sally Thorne, Vancouver, British Columbia, Canada

Judith Wuest, Fredericton, New Brunswick, Canada

$7 \mathrm{o}$ the individuals who helped us in the selection of quantitative studies that were clinically important and relevant 1 to practising nurses in their areas of expertise:

Heather Arthur, Hamilton, Ontario, Canada Wendy Goodine, Etobicoke, Ontario, Canada

Ellen McDonald, Hamilton, Ontario, Canada

Janet Pinelli, Hamilton, Ontario, Canada

To Brian Hutchison, who provided thoughtful methodological advice about selected studies, and to Geoff Norman 1 who assisted with the formulation of definitions included in the glossary.

nd finally, to the people who served as peer reviewers for the EBN Notebook and Implementation Forum, for their Athoughtful and timely feedback:

Jose Closs, Leeds, UK

Jeannette LeGris, Hamilton, Ontario, Canada

Rob Newell, Leeds, UK

Shahirose Premji, Hamilton, Ontario, Canada

Joan Royle, Hamilton, Ontario, Canada
Bonnie Stevens, Toronto, Ontario, Canada

David Torgerson, York, UK

Carl Thompson, York, UK

Judy Wuest, Fredericton, New Brunswick, Canada

$A \mathrm{n}$ important step in the practice of evidence-based nursing is the sharing of successful implementation strategies. We therefore wel-

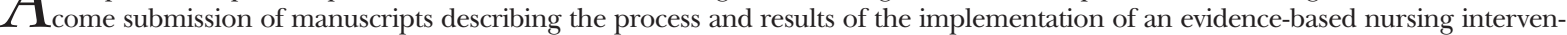
tion. Manuscripts should be no longer than 1500 words, including references. All manuscripts will be peer reviewed and submission does not guarantee publication.

We also welcome letters from our readers about Evidence-Based Nursing. We would like to hear about the positive and negative aspects of our journal. Your feedback is most important in assisting us to produce a high quality journal which is useful to the practising nurse. Letters should be no longer than 400 words.

All submitted material should be typewritten, double spaced, and mailed or faxed to Nicky Cullum in the UK editorial office (Centre for Evidence Based Nursing, University of York, Genesis 6, York Science Park, York YO10 5DQ. Fax: + 44 (0) 1904 434102). 